\title{
A BeppoSAX view of transient black hole candidates in quiescence
}

\author{
S. Campana ${ }^{1}$, A. N. Parmar ${ }^{2}$, and L. Stella ${ }^{3}$ \\ 1 Osservatorio Astronomico di Brera, Via E. Bianchi 46, 23807 Merate (Lecco), Italy \\ 2 Astrophysics Division, Space Science Department of ESA, ESTEC, PO Box 299, 2200 AG Noordwijk, \\ The Netherlands \\ 3 Osservatorio Astronomico di Roma, Via Frascati 33, 00040 Monteporzio Catone (Roma), Italy
}

Received 5 March 2001 / Accepted 2 April 2001

\begin{abstract}
We report BeppoSAX observations of five transient black hole candidates during their quiescent phase. We confirm that these sources are $\mathrm{X}$-ray faint, improving on and complementing existing upper limits. We derive 1-10 keV upper limits for GRO J0422+32, GRS 1009-45, 4U 1630-47 and XTE J1748-288, which range from $2 \times 10^{32} \mathrm{erg} \mathrm{s}^{-1}$ to $5 \times 10^{34} \mathrm{erg} \mathrm{s}^{-1}$. We positively detect GS $2023+338$. Its X-ray spectrum can be fit by a power law (photon index $\Gamma=1.9$ ) or a thermal bremsstrahlung (with $k T_{\mathrm{br}}=7 \mathrm{keV}$ ), converting in both cases to a $1-10 \mathrm{keV}$ unabsorbed luminosity of $\sim 10^{33} \mathrm{erg} \mathrm{s}^{-1}$. These values are comparable to the ones derived during an ASCA observation in 1994, indicating that the source remains stable over a $\sim 5$ yr baseline.
\end{abstract}

Key words. binaries: general - black hole physics - X-rays: stars

\section{Introduction}

Transient black hole binaries are characterised by bright outbursts lasting up to several months and recurring every 1-100 yr (with a clear preference for long recurrence times). In between these outbursts, sources are quiescent with very low $\mathrm{X}$-ray luminosities. At the present time only five transient Black Hole Candidates (BHCs) have been detected in quiescence and upper limits exist for six other systems (Asai et al. 1998; Menou et al. 1999a; Campana \& Stella 2000; Garcia et al. 2001).

A $0620-00$ was the first BHC to be detected in quiescence. Despite the small number of photons collected in the ROSAT observation the spectrum was determined to be soft. A $0.5-10 \mathrm{keV}$ luminosity of $L_{\mathrm{X}} \sim 10^{31} \mathrm{erg} \mathrm{s}^{-1}$ (for a distance of $d=1.2 \mathrm{kpc}$ ) was obtained by extrapolating the ROSAT data (McClintock et al. 1995). Chandra detected the source at a level $\sim 10$ times lower (Garcia et al. 2001). GS 2023+338 (V 404 Cyg) was observed with ASCA at a $0.5-10 \mathrm{keV}$ luminosity of $L_{\mathrm{X}} \sim 2 \times 10^{33} \mathrm{erg} \mathrm{s}^{-1}$ $(d=3.5 \mathrm{kpc} ;$ Narayan et al. 1997). The spectrum is well fit by a power law with photon index $\Gamma \sim 2.1_{-0.3}^{+0.5}$ or by a thermal bremsstrahlung with an equivalent temperature of $k T_{\mathrm{br}}=4.6_{-1.5}^{+3.6} \mathrm{keV}$. Analysing the same dataset Asai et al. (1998) obtained $\Gamma=1.7_{-0.2}^{+0.3}$. Finally, GRO J1655-40

Send offprint requests to: S. Campana, e-mail: campana@merate.mi.astro.it was detected again by ASCA at $L_{\mathrm{X}} \sim 2 \times 10^{32} \mathrm{erg} \mathrm{s}^{-1}$ $(d=3.2 \mathrm{kpc}$; Hameury et al. 1997). The spectrum could be described by a power law model with a quoted in$\operatorname{dex} \Gamma \sim 1.5 \pm 0.6$ (Hameury et al. 1997). Asai et al. (1998) analysed the same dataset and found $\Gamma=0.7_{-0.4}^{+2.1}$. Chandra observed this source when it was a factor of $\sim 10$ lower (Garcia et al. 2001).

Since the discovery of their low X-ray quiescent luminosity, the study of BHCs in quiescence has attracted attention because this emission might be able to distinguish them from quiescent neutron star systems (for a review see Campana et al. 1998a). At the very beginning, Tanaka \& Shibazaki (1996) argued that BHCs and neutron stars systems at low luminosities show similar soft spectra, well approximated by a single temperature black body $\left(k T_{\mathrm{bb}} \sim 0.2-0.3 \mathrm{keV}\right)$. This was based on (and biased by) ROSAT results and only on a single BHC (A 0620-00). ASCA and BeppoSAX observations led to the discovery of hard tails in neutron star systems (Asai et al. 1996, 1998; Campana et al. 1998b, 2000) as well as the detection of non-thermal spectra in GS 2023+338 and GRO J1655-40, challenging this picture (however, GS 2023+338 and GRO J1655-40 have orbital periods longer than the great majority of low mass transients and, therefore, likely experience a higher time-averaged mass exchange rate). In a recent paper, Garcia et al. (2001) reported on deep Chandra observations of black hole 
Table 1. Summary of BeppoSAX observations.

\begin{tabular}{ccccc}
\hline Source & $\begin{array}{c}\text { LECS Exp. time } \\
(\mathrm{s})\end{array}$ & $\begin{array}{c}\text { MECS Exp. time } \\
(\mathrm{s})^{*}\end{array}$ & $\begin{array}{c}\text { PDS Exp. Time } \\
(\mathrm{s})\end{array}$ & $\begin{array}{c}\text { Observation } \\
\text { number }\end{array}$ \\
\hline GRO J0422+32 & 19681 & $46274(2)$ & 21275 & 20535001 \\
GRS 1009-45 & 19773 & $40675(2)$ & 20689 & 20607001 \\
4U 1630-47 & 13357 & $32944(3)$ & 22426 & 20315001 \\
GS 2023+338 & - & $21384(3)$ & 10098 & 20303001 \\
XTE J1748-288 & 19174 & $47884(2)$ & 34787 & 20130002 \\
\hline
\end{tabular}

* In parenthesis the number of MECS active units.

+ Source at $5^{\prime}$ off-axis.

transients in quiescence, detecting two new sources with short orbital period (GRO J0422+32 and GS 2000+25). Now three short orbital period BHCs have been detected all with $0.5-10 \mathrm{keV}$ luminosities in the $2-8 \times 10^{30} \mathrm{erg} \mathrm{s}^{-1}$ range. These data confirm that black hole transients in quiescence are much less luminous than the corresponding neutron star transient systems in $X$-rays.

Advection dominated accretion flow (ADAF) models have become popular to explaining the low luminosity of quiescent BHCs as well as their spectral energy distribution (Narayan et al. 1996; Narayan et al. 1997; Menou et al. 1999a, 1999b). In these models, a large fraction of the gravitational energy is advected into the black hole, therefore lowering considerably the radiative efficiency of the accretion process. Observations show that the ratio of minimum $\mathrm{X}$-ray luminosity in quiescence to maximum $\mathrm{X}$-ray luminosity in outburst is significantly smaller (a factor of about 100) in black hole transients than in and neutron star transients (Narayan et al. 1997; Garcia et al. 1998; Garcia et al. 2001). Campana \& Stella (2000) noted that in the latest ADAF models the optical/UV luminosity derives from synchrotron radiation produced by the ADAF itself and therefore must be included in the luminosity budget. Ascribing the bulk of the residual optical/UV flux to the ADAF removes much of the difference in luminosity swing between black hole and neutron star transients, weakening one of the main drives of ADAF models. Possible ways out are that a large fraction of the accreting matter is stopped/evaporated at the outer disk boundary with only a very small fraction leaking towards the compact object and/or ADAFs do not contribute to the optical luminosity as in the older ADAF models (Campana \& Stella 2000).

A different interpretation relies on the coronal activity of the companion star. This mechanism provides a quiescent luminosity of the order of $10^{29}-10^{30} \mathrm{erg} \mathrm{s}^{-1}$ for main sequence stars (Eracleous et al. 1991), unless subgiant companions are present $\left(10^{31}-10^{32} \mathrm{erg} \mathrm{s}^{-1}\right.$ for RS CVn systems; Campana \& Stella 2000; Bildsten \& Rutledge 2000). This mechanism therefore might be at work only in the most X-ray faint BHCs.

In this paper we present a comprehensive view of black hole transients in quiescence observed with the Italian/Dutch satellite BeppoSAX complementing and improving current upper limits and existing spectra. In Sect. 2 we describe the data set and analysis. In Sect. 3 we discuss the results.

\section{Observations and data reduction}

We present the results of the observations of black hole transients in quiescence carried out with the BeppoSAX satellite (Boella et al. 1997a). We analysed the data from the Low Energy Concentrator Spectrometer (LECS; 0.1-10 keV, Parmar et al. 1997a) and the Medium Energy Concentrator Spectrometer (MECS; 1.3-10 keV, Boella et al. 1997b). As usual, LECS data were collected only during satellite night-time leading to shorter exposure times. Upper limits in the hard X-ray band were obtained with the Phoswich Detector System (PDS, 15-300 keV; Frontera et al. 1997). The PDS collimators rocked on and off the target in order to monitor the background. This also resulted in a shorter exposure time than the MECS.

BeppoSAX observed four BHCs in quiescence: GRO J0422+32, GRS 1009-45, 4U 1630-47 and GS 2023+338. XTE J1748-288 was serendipitously observed during a pointing toward the Galactic Center region. For a summary of the observations see Table 1.

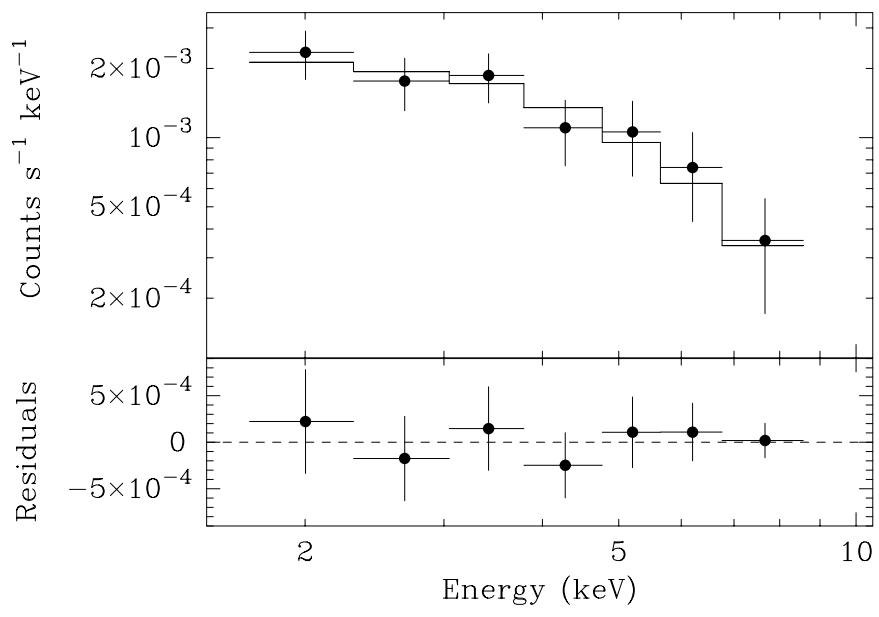

Fig. 1. X-ray spectrum of GS $2023+338$ in quiescence. The spectrum is fit with an absorbed power law model. In the lower panel the ratio between the data and the model is presented. 
The BeppoSAX images were searched for sources at the position of the BHCs' optical counterpart. Only one out of five BHCs was detected (GS 2023+338), confirming the elusiveness of this kind of source in quiescence.

Upper limits for imaging instruments were determined counting the number of events within a specified box ( $4^{\prime}$ radius), correcting those counts for vignetting, exposure and point spread function and comparing them with the number of events in an outer background box free of sources. In the case of the PDS collimators source upper limits were derived by comparing the number of events collected on and off the the source.

GRO J0422+32 was observed by BeppoSAX on September 2, 1998, about 2200 days after the previous outburst. The source was not detected and we place a $3 \sigma$ upper limit of $2 \times 10^{-3} \mathrm{c} \mathrm{s}^{-1}$ on the MECS count rate, which translates to a flux limit of $1.5 \times 10^{-13} \mathrm{erg} \mathrm{s}^{-1} \mathrm{~cm}^{-2}$ $(2-10 \mathrm{keV})$, assuming a power law spectrum with photon index $\Gamma=2.1$ (the same as observed in GS $2023+338$ in quiescence; Narayan et al. 1997) and the galactic column density in the direction of the source $N_{\mathrm{H}}=1.7 \times 10^{21} \mathrm{~cm}^{-2}$. For a distance of $3.6 \mathrm{kpc}$ (Shrader et al. 1994) we obtain an upper limit on the $2-10 \mathrm{keV}$ luminosity of $2 \times 10^{32} \mathrm{erg} \mathrm{s}^{-1}$. This is to be compared with the value derived by Garcia et al. (1998) of $4 \times 10^{31} \mathrm{erg} \mathrm{s}^{-1}$ $(0.5-10 \mathrm{keV})$ which was obtained by extrapolating the result of a deep ROSAT-HRI pointing over a much larger energy range. The BeppoSAX PDS data give an upper limit of $\sim 6 \times 10^{-12} \mathrm{erg} \mathrm{s}^{-1} \mathrm{~cm}^{-2}$ in the $15-50 \mathrm{keV}$ energy band, which translates to $\sim 9 \times 10^{33} \mathrm{erg} \mathrm{s}^{-1}$.

4U 1630-47 was observed on March 26, 1997, about one year after the 1996 outburst (this source in contrast with other transient BHCs shows recurrent outbursts, Parmar et al. 1995). The $3 \sigma$ upper limit on the MECS count rate amounts to $1.2 \times 10^{-3} \mathrm{c} \mathrm{s}^{-1}$. This translates to an upper limit on the $2-10 \mathrm{keV}$ unabsorbed luminosity of $2 \times 10^{33} \mathrm{erg} \mathrm{s}^{-1}$ for a distance of $10 \mathrm{kpc}$, a power law with $\Gamma=2.1$ and $N_{\mathrm{H}}=8 \times 10^{22} \mathrm{~cm}^{-2}$ (Oosterbroek et al. 1998). Even if this upper limit is in the range of those previously obtained with the ROSATHRI $\left(8 \times 10^{33} \mathrm{erg} \mathrm{s}^{-1} 0.2-2.4 \mathrm{keV}\right)$ and ROSAT-PSPC $\left(7 \times 10^{32} \mathrm{erg} \mathrm{s}^{-1} 0.2-2.4 \mathrm{keV}\right.$; Parmar et al. 1997b), the more extended energy range of the MECS makes the BeppoSAX limit less affected by interstellar absorption. At high energies, the PDS limit on the $15-50 \mathrm{keV}$ flux was of $\sim 9 \times 10^{-11} \mathrm{erg} \mathrm{s}^{-1} \mathrm{~cm}^{-2}$ (corresponding to $\sim 1 \times$ $10^{36} \mathrm{erg} \mathrm{s}^{-1}$ ). This relatively high value is due to the presence of a number of sources in the PDS field of view, which are clearly seen in the MECS.

GRS 1009-45 was observed on December 3, 1998, about 1900 days after the previous outburst. We obtain a $3 \sigma$ upper limit of $1.6 \times 10^{-3} \mathrm{c} \mathrm{s}^{-1}$ on the MECS count rate. This translates into an upper limit of $1 \times 10^{33} \mathrm{erg} \mathrm{s}^{-1}$ on the $2-10 \mathrm{keV}$ unabsorbed luminosity for a distance of $3 \mathrm{kpc}$ (Della Valle et al. 1997), a power law with $\Gamma=2.1$ and $N_{\mathrm{H}}=10^{21} \mathrm{~cm}^{-2}$. The $15-50 \mathrm{keV}$ upper limit from the PDS amounts to $\sim 7 \times 10^{33} \mathrm{erg} \mathrm{s}^{-1}$. No previous upper limits exist on the $2-10 \mathrm{keV}$ and $15-50 \mathrm{keV}$ luminosities.
XTE J1748-288 was observed on April 5, 1997, about $400 \mathrm{~d}$ before its discovery. We obtain a $3 \sigma$ upper limit on the MECS count rate of $6 \times 10^{-3} \mathrm{c} \mathrm{s}^{-1}$. Despite the relatively long exposure, this fairly high value is due to the source position right on the MECS "strongback" (Boella et al. 1997b). Using the appropriate response matrix we derive a flux of $<6 \times 10^{-12} \mathrm{erg} \mathrm{s}^{-1} \mathrm{~cm}^{-2}$ (i.e. $<5 \times 10^{34} \mathrm{erg} \mathrm{s}^{-1}$ at $8.5 \mathrm{kpc}$ ). No upper limits in the 15-50 keV energy band can be set due to the presence of strong sources in the field.

In passing we note that GX 339-4 has been observed and detected by BeppoSAX at a level of $\sim 6 \times 10^{33} \mathrm{erg} \mathrm{s}^{-1}$ (Kong et al. 2000). This luminosity level is high when compared with the other BHCs, suggesting that the observed "off"-state of GX 339-4 may not correspond to the quiescent state of a transient system (note also that GX 339-4 is usually considered a persistent source). Another interesting system monitored by BeppoSAX is CI Cam (XTE J0421+560). This source was observed three times in quiescence (156, 541 and $690 \mathrm{~d}$ after the latest outburst, respectively) with very different outcomes: $i)$ a very soft spectrum $(\Gamma \sim 4)$ with a low column density $\left(N_{\mathrm{H}} \sim 10^{21} \mathrm{~cm}^{-2}\right)$ and a 1-10 keV luminosity of $2 \times 10^{32} \operatorname{erg~s}^{-1}$ (at $2 \mathrm{kpc}$ ); ii) a hard spectrum $(\Gamma \sim 2)$ with a high column density $\left(N_{\mathrm{H}} \sim 4 \times 10^{23} \mathrm{~cm}^{-2}\right)$ and a luminosity of $4 \times 10^{33} \mathrm{erg} \mathrm{s}^{-1}$; iii) undetected with an upper limit of $4 \times 10^{32} \mathrm{erg} \mathrm{s}^{-1}$ (Parmar et al. 2000). However, the nature of the compact object of CI Cam is presently unclear.

\subsection{Spectral analysis: GS $2023+338$}

The source spectrum of GS $2023+338$ was extracted from the MECS data within a radius of $4^{\prime}$ centered on the source position (LECS data were unavailable). We collected 433 photons within the full energy range. Background subtraction was performed using the standard background files. We used the publicly available calibration files at 2000 January and XSPEC 11.0. We rebinned the MECS spectrum in order to have at least 50 photons per spectral bin, resulting in seven bins. The lack of the LECS data forced us to adopt a value for the absorbing column density. Following Wagner et al. (1994) and Narayan et al. (1997) we used a value of $N_{\mathrm{H}}=10^{22} \mathrm{~cm}^{-2}$. With this value of the column density a power law model provides an adequate description of the data with a photon index $\Gamma=1.9_{-0.5}^{+0.6}$ (errors at $90 \%$ confidence level for one parameter of interest) with a $\chi_{\text {red }}^{2}=0.2$ (for 5 d.o.f.). A bremsstrahlung model provides also a good fit with $k T_{\mathrm{br}}=6.9_{-3.7}^{+31.5} \mathrm{keV}$ and $\chi_{\text {red }}^{2}=0.3$. A black body model instead fails to successfully describe the spectra with $\chi_{\text {red }}^{2}=1.4\left(k T_{\mathrm{bb}}=\right.$ $1.0_{-0.2}^{+0.3} \mathrm{keV}$ ). This last model can be reconciled with the data only by assuming a very low column density (formally null $)$ and $k T_{\mathrm{bb}}=1.1_{-1.1}^{+0.1} \mathrm{keV}\left(\chi_{\text {red }}^{2}=1.1\right)$. The unabsorbed $1-10 \mathrm{keV}$ flux as derived with the power law model amounts to $7.1 \times 10^{-13} \mathrm{erg} \mathrm{s}^{-1} \mathrm{~cm}^{-2}$. This is to be compared with the $8.2 \times 10^{-13} \mathrm{erg} \mathrm{s}^{-1} \mathrm{~cm}^{-2}$ value derived 
from the ASCA observation (Narayan et al. 1997). The 1$10 \mathrm{keV}$ unabsorbed luminosity is $L_{\mathrm{X}}=1.2 \times 10^{33} \mathrm{erg} \mathrm{s}^{-1}$ (at $3.5 \mathrm{kpc}$ ). The PDS provides an upper limit to the 15-50 keV luminosity of $\sim 3 \times 10^{34} \mathrm{erg} \mathrm{s}^{-1}$.

\section{Conclusions}

We report on the BeppoSAX view of transient BHCs in quiescence. We further confirm that BHCs in quiescence are $\mathrm{X}$-ray faint with luminosities below $\sim 10^{32}-10^{33} \mathrm{erg} \mathrm{s}^{-1}$ (at which neutron star transients are usually detected) and report for the first time upper limits in the $2-10 \mathrm{keV}$ energy band for four sources: GRO J0422+32, GRS 1009-45, 4U 1630-47 and XTE J1748-288. These limits improve and confirm existing extrapolations from lower energy bands (mainly ROSAT, $0.1-2.4 \mathrm{keV}$ ). Moreover, we quote the upper limits in the hard energy band 15-50 keV derived from the PDS data. These hard X-ray luminosity limits might be useful to constrain ADAF models, for which high energy tails are expected (current models easily satisfy these constraints, e.g. Menou et al. 1999a). After the failure of ASTRO-E, these limits will remain unrivaled for years to come.

One exception to the above cases, that was confirmed also by BeppoSAX observation, is GS $2023+338$, which revealed a level of $\sim 10^{33} \mathrm{erg} \mathrm{s}^{-1}(1-10 \mathrm{keV})$. The GS $2023+338$ luminosity derived is at a level and with a spectrum similar to those of the ASCA observation five years before (Asai et al. 1998; Hameury et al. 1997). This indicates that, within the uncertainties, the spectrum and the flux level of GS 2023+338 in quiescence remains fairly constant.

Acknowledgements. We thank T. Mineo for making available to us off-axis MECS response matrices. This research has made use of SAXDAS linearised and cleaned event files (Rev.2.0) produced at the BeppoSAX Science Data Center. This work was partially supported through ASI grants.

\section{References}

Asai, K., et al. 1996, PASJ, 48, 257

Asai, K., et al. 1998, PASJ, 50, 611

Bildsten, L., \& Rutledge, R. E. 2000, ApJ, 541, 908

Boella, G., et al. 1997a, A\&AS, 122, 299

Boella, G., et al. 1997b, A\&AS, 122, 327

Campana, S., \& Stella, L. 2000, ApJ, 541, 849

Campana, S., Stella, L., Mereghetti, S., \& Cremonesi, D. 2000, A\&A, 358, 583

Campana, S., et al. 1998a, A\&AR, 8, 269

Campana, S., et al. 1998b, ApJ, 499, L65

Della Valle, M., Benetti, S., Cappellaro, E., \& Wheeler, C. 1997, A\&A, 318, 179

Eracleous, M., Halpern, J., \& Patterson, J. 1991, ApJ, 382, 290

Frontera, F., et al. 1997, A\&AS, 122, 357

Garcia, M. R., McClintock, J. E., Narayan, R., \& Callanan, P. J. 1998, in Proceedings of the 13th North American Workshop on CVs, ed. S. Howell, E. Kulkeers, \& C. Woodward, San Francisco ASP, 506

Garcia, M. R., et al. 2001, submitted to ApJ [astro-ph/0012452]

Hameury, J.-M., Lasota, J.-P., McClintock, J. E., \& Narayan, R. 1997, ApJ, 489, 234

Kong, A. K. H., Kulkeers, E., Charles, P. A., \& Homer, L. 2000, MNRAS, 312, L49

McClintock, J. E., Horne, K., \& Remillard, R. A. 1995, ApJ, 442,358

Menou, K., Narayan, R., \& Lasota, J.-P. 1999a, ApJ, 513, 811

Menou, K., et al. 1999b, ApJ, 520, 276

Narayan, R., Barret, D., \& McClintock, J. E. 1997, ApJ, 482, 448

Narayan, R., McClintock, J. E., \& Yi, I. 1996, ApJ, 457, 821

Oosterbroek, T., et al. 1998, A\&A, 340, 431

Parmar, A. N., Angelini, L., \& White, N. E. 1995, ApJ, 452, 129

Parmar, A. N., et al. 1997a, A\&AS, 122, 309

Parmar, A. N., et al. 1997b, A\&A, 319, 855

Parmar, A. N., et al. 2000, A\&A, 360, L31

Shrader, C. R., et al. 1994, ApJ, 434, 698

Tanaka, Y., \& Shibazaki, N. 1996, ARA\&A, 34, 607

Wagner, R., et al. 1994, ApJ, 429, L25 